\title{
Announcement
}

\section{Special Issue in Honour of David Montgomery}

The plasma physics community has reacted enthusiastically to the Journal's invitation to submit papers to a special issue to mark David Montgomery's 60th birthday - so much so that the special issue will be published in two parts, in December 1996 and January 1997. A large number of associates and admirers of Professor Montgomery have submitted papers, mainly on nonlinear plasma physics, which is, of course, his special field of interest. These papers will provide a comprehensive account of recent developments, and should be of great value to all researchers with an interest in the nonlinear behaviour of plasmas.

Alan Cairns 